\title{
N. Eilan, Ch. Hoerl, T. McCormack et J. Roessler (dir.), Joint attention : communication and other minds. Issues in philosophy and psychology
}

Oxford, Clarendon Press, 2005

\section{Natalie Depraz}

\section{OpenEdition}

Journals

Édition électronique

URL : http://journals.openedition.org/alter/1757

DOI : $10.4000 /$ alter. 1757

ISSN : 2558-7927

Éditeur :

Association ALTER, Archives Husserl (CNRS-UMR 8547)

Édition imprimée

Date de publication : 1 octobre 2010

Pagination : 333-338

ISBN : 2-9522374-6-8

ISSN : $1249-8947$

\section{Référence électronique}

Natalie Depraz, « N. Eilan, Ch. Hoerl, T. McCormack et J. Roessler (dir.), Joint attention : communication and other minds. Issues in philosophy and psychology ", Alter [En ligne], 18 | 2010, mis en ligne le 01 juin 2020, consulté le 25 septembre 2020. URL : http://journals.openedition.org/alter/1757 ; DOI : https:// doi.org/10.4000/alter.1757 


\title{
N. EILAN ${ }^{1}$, CH. HOERL, T. MCCORMACK ET J. ROESSLER \\ (DIR.), JOINT ATTENTION : COMMUNICATION AND OTHER MINDS. ISSUES IN PHILOSOPHY AND PSYCHOLOGY, OXFORD, CLARENDON PRESS, 2005
}

\begin{abstract}
Natalie Depraz
Dans sa Préface, Naomi Eilan rappelle la motivation première du présent volume: il s'agissait de répondre à la question de l'importance et du rôle joué par la "cognition sociale" dans la constitution objective des représentations spatiales, objet d'un volume antérieur, lesquelles se trouvaient alors appréhendées sur un mode essentiellement solipsiste. Cette problématique générale peut paraître bien éloignée de la thématique retenue pour ce volume, «l'attention conjointe », mais on va voir qu'en réalité, cette dernière est au cœur du problème posé. De plus, le fait de situer la question dans le cadre de la « cognition sociale » et de son rôle pour l'objectivité de la connaissance permet de montrer immédiatement combien les questions que s'est posé Husserl dès le début du vingtième siècle et durant les trois premières décennies se situent très exactement dans cette perspective. En
\end{abstract}

1. Spatial Representation: Problems in Philosophy and Psychology (ed. with R. McCarthy \& W. Brewer), Oxford University Press, 1999; The Body and the Self (ed. with J. Bermudez \& A. Marcel), MIT Press, 1995 ; "The First Person Perspective", Proceedings of the Aristotelian Society, 1996 ; "Objectivity and the Perspective of Consciousness", European Journal of Philosophy, 1997 ; "Perceptual Intentionality, Attention and Consciousness", in A. O'Hear, ed.: Contemporary Issues in the Philosophy of Mind, Cambridge University Press, 1998; "On Understanding Schizophrenia", in D. Zahavi, ed.: Exploring the Self: Philosophical and Psychopathological Perspectives on Self-Experience, John Benjamins, 2000 ; 'Meaning, Truth, and the Self', Philosophy, Psychiatry and Psychology 8, 2001, p. 121-132; Agency and Self Awareness (ed. with J. Roessler), Oxford University Press, 2003 ; The Reality of Consciousness, Oxford University Press, 2005. Voilà quelques titres d'ouvrages et d'articles de N. Eilan, qui enseigne à l'Université de Warwick et y a mis en place un projet de recherche intitulé «Consciousness and Selfconsciousness. Issues in Philosophy and Psychology» dans le cadre duquel est paru le livre que je recense présentement. 


\section{L'attention}

effet, l'intérêt du phénoménologue pour l'intersubjectivité s'inscrit dans le cadre d'une recherche centrée sur la connaissance des objets et le statut de l'objectivité en général. Dès lors, ce que l'on nomme "l'expérience d'autrui » et le statut de l'empathie n'est pas abordé, c'est bien connu, du point de vue de sa teneur émotionnelle ou en relation avec une problématique éthique, comme M. Scheler le fera exemplairement, mais eu égard à l'objectivité de la connaissance que peut me permettre d'acquérir l'autre en tant qu'autre sujet, ainsi que les autres en tant que membres d'un monde commun. En ce sens, ce que les philosophes anglo-saxons nomment ici social cognition (relevons justement l'accent porté sur la connaissance dans l'usage du terme « cognition »!) répond très précisément à la recherche husserlienne du type de connaissance renouvelée permise par l'introduction d'autrui et des autres. Dans les deux cas, le but est l'exploration d'une forme d'objectivité de la connaissance où les autres se voient jouer un rôle déterminant.

Ceci étant posé, la voie empruntée par N. Eilan et ses collaborateurs pour explorer ce rôle de l'intersubjectivité dans la constitution de l'objectivité apparaît dans toute son originalité. En effet, l'attention conjointe est un phénomène qui intervient précocement dans le développement de l'enfant, autour de 12 mois selon l'hypothèse-standard en psychologie de l'enfant, ${ }^{2}$ et qui met en selle dès le départ, du sein d'une interaction sociale immédiate, la possibilité très générale de la pensée en lien avec l'idée d'un monde objectif. Ainsi, le cadre proposé moyennant l'attention constitue une alternative extrêmement féconde à la proposition husserlienne d'une constitution de l'objectivité par le moyen de l'expérience associative-passive et imageante de l'empathie. En effet, il aura fallu à Husserl une refonte de l'empathie qui la débarrasse de ses bases esthétiques et émotionnelles et la resitue dans un cadre strictement gnoséologique (ce qui est tout à la fois l'originalité et le tour de force du fondateur de la phénoménologie) pour pouvoir en faire le pivot de l'articulation entre intersubjectivité et objectivité. La piste que suit dans ce volume l'équipe de $\mathrm{N}$. Eilan consiste à faire droit à ce phénomène essentiellement d'emblée cognitif de l'attention conjointe à titre de pivot adéquat à une telle articulation entre intersubjectivité et objectivité.

À mesure que nous allons recenser certaines des contributions les plus centrales dans cette perspective, nous voudrions évaluer l'intérêt et l'originalité de cette hypothèse, y compris dans le dialogue avec la phénoménologie husserlienne et merleau-pontienne. En effet, les articles que nous allons privilégier manifestent une ouverture pour la tradition phénoménologique et y cherchent également des ressources tout à la fois expérientielles et conceptuelles.

2. À ce propos, les travaux plus récents de $\mathrm{H}$. Tremblay et de $\mathrm{K}$. Rovira tendent à montrer que ce phénomène apparaît beaucoup plus tôt, et qu'il est le fait d'une interaction non pas duelle mais plurielle. Cf. H. Tremblay \& K. Rovira (Université de Rouen, Laboratoire PSY.CO EA 1780), « Joint visual attention and social triangular engagement at 3 and 6 months », Infant Behavior $\mathcal{E}$ Development 30 (2007), p. 366-379. 
Je me centrerai pour ce faire et pour commencer sur la longue Introduction très détaillée de $\mathrm{N}$. Eilan à l'ensemble du volume, qui présente précisément en quoi consiste concrètement le phénomène de l'attention conjointe, ainsi que les deux théories majeures (rationaliste/émotionnelle) qui s'opposent quant à la compréhension de ce phénomène. Puis, je $\mathrm{m}^{\prime}$ attacherai aux deux articles à mon sens les plus représentatifs de chacune de ces deux théories, celui de Joseph Call et Michael Tomasello et celui de R. Peter Hobson. Pour finir, j'en viendrai à l'article de Johannes Roessler, qui présente à mon sens le plus d'affinité avec une approche phénoménologique possible de ce phénomène.

\section{Qu'est-ce que l'attention conjointe? «Mutual awareness"}

Dans son Introduction générale au volume, N. Eilan présente de façon très claire et très simple ce à quoi correspond le phénomène de l'attention conjointe. Autant citer pour commencer le début de son texte, plutôt que de redire moins bien ce qu'elle dit si bien: "Quelque part autour de leur premier anniversaire, la plupart des enfants commencent à rentrer dans des segments relativement soutenus de co-présence aux objets de leur environnement, et ce, avec la personne qui s'occupe d'eux. Autour de 18 mois, dans de nombreux comptes-rendus, ils sont engagés dans des épisodes complets d'attention conjointe. En suivant la façon qu'ont les psychologues développementaux d'employer le terme, pour qu'il y ait attention conjointe, il n'est pas suffisant que l'enfant et l'adulte soient en fait présents au même objet, ni que l'attention de l'un soit la cause de l'attention de l'autre. Ce dernier processus peut se produire et se produit effectivement beaucoup plus tôt, à chaque fois que l'adulte suit le regard de l'enfant et se fixe sur le même objet que lui, alors que le bébé y est présent ; ou bien, à partir de l'âge de 6 mois, lorsque les bébés commencent à suivre le regard de l'adulte. Il n'y a cas pertinent d'attention conjointe que lorsque la présence commune de l'adulte et de l'enfant au même objet est, pour employer l'expression de Sperber et Wilson dans un livre de 1986 (Relevance: communication and cognition), "mutuellement manifeste". Certains psychologues parlent parfois d'une telle "conjonction" (jointness) comme d'un cas d'attention "partagée" (shared) par l'enfant et l'adulte, ou comme un cas $\mathrm{d}^{\prime \prime}$ esprits qui se rencontrent" (meeting of minds), toutes ces expressions ayant pour but de formuler l'idée selon laquelle, quand il y a attention conjointe, tout ce qui concerne le fait que les deux sujets sont présents au même objet est ouvertement livré au regard des deux participants, est manifeste et explicite pour les deux ${ }^{3}$. » Il s'agit donc d'un phénomène pré-verbal, initiateur d'une socialité immédiate, et qui témoigne d'une forme de conscience particulière 


\section{L'attention}

à l'événement : ce n'est pas une conscience qui émerge comme conscience de soi depuis soi-même, individuellement, mais elle est originairement " relationnelle », c'est-à-dire structurée par la conscience même de l'autre au même événement. Pour caractériser une telle conscience, N. Eilan (et elle n'est la seule, bien sûr !) parle de mutual awareness : une conscience mutuelle ${ }^{4}$. Plus loin, elle précise que cette présence à l'autre devant l'objet est vécue par l'autre sur le même mode que je le vis moi-même, et c'est pourquoi elle introduit l'expression caractéristique de «joint attention triangle ${ }^{5}$ ». À cet égard, elle s'appuie amplement sur l'ouvrage de D. Davidson, "The emergence of thought » in Subjective, Intersubjective, Objective (OUP, 2001) de façon à montrer comment la trinagulation en jeu est l'origine même de la socialité ${ }^{6}$.

\section{Deux théories en présence}

C'est ce qui la conduit à proposer une brève ébauche des deux théories en présence sur ce phénomène 7 . N. Eilan distingue une théorie "mentaliste » de l'attention conjointe, représentée pour l'essentiel par le travail de Tomasello ${ }^{8}$, lequel montre comment l'enfant, entre 9 et 18 mois, entre dans une révolution "cognitive », et une théorie "émotionnelle », portée initialement par Werner et Kaplan ${ }^{9}$, lesquels présentent l'attention conjointe comme une forme de régulation inter-affective.

Plus avant, la position de Tomasello est décrite en termes de «coopération rationnelle » : le mécanisme de l'attention conjointe correspondrait à la rencontre de deux activités intentionnelles causales ; à cela s'opposent des conceptions portées par des auteurs comme Campbell ou, exemplairement dans le présent volume, Hobson, soit qu'ils situent l'attention au niveau d'un mécanisme primitif d'orientation, de type sub-personnel lisible sur un plan neuronal (Campbell) ${ }^{10}$, soit qu'ils le formulent à un niveau subjectif vécu, appréhendé en termes d'ouverture co-affective (Hobson) ${ }^{11}$. On a affaire en réalité à trois modèles explicatifs distincts, l'un de type réflexifrationnel, l'autre de type sub-conscient/neuronal, le troisième, enfin, de type affectif-émotionnel. Pour des raisons d'économie, je me centrerai ici sur les

4. Op. cit., p. 2.

5. Op. cit., p. 5.

6. Op. cit., p. 7-10.

7. Op. cit., p. 16-18.

8. M. Tomasello, "Joint attention and social cognition », in C. Moore and P. Dunham (eds.), Joint Attention: Its origin and rôle in development, Hillsdale, NJ, 1995, Erlbaum, p. 103-130; The Cultural Origin of Human Cognition, Harvard, Cambridge Mass., 1999.

9. H. Werner \& B. Kaplan, Symbol Formation, Hillsdale, NJ : Erlbaum, 1963.

10. J. Campbell, «Joint Attention and Common Knowledge » N. Eilan et al., eds., op. cit., p. 287398.

11. P. Hobson, « What Puts the Jointness into Joint Attention », N. Eilan ed., op. cit., p. 185-205. 
positions contrastées de Tomasello et de Hobson, qui représentent les positions $n^{\circ} 1$ et $n^{\circ} 3$, et je laisse de côté le modèle explicatif neuronal, plus distant d'une intelligibilité phénoménologique, rationnelle ou émotionnelle.

Dans «What Chimpanzees Know about Seeing, Revisited : An Explanation of the Third Kind ${ }^{12}$ », Joseph Call and Michael Tomasello utilisent le contreexemple des chimpanzés (selon eux, c'est aussi le cas des enfants autistes) pour valider leur hypothèse de la coordination « rationnelle " standard à l'œuvre dans le mécanisme de l'attention conjointe. Ils détaillent, sur le cas du comportement des chimpanzés, une capacité comportementale qui ne passe pas par une rationalité cognitive liée à une activité mentale: «les chimpanzés (...) nous montrent la manière la plus intelligente dont un individu peut comprendre et inter-agir avec ses congénères en l'absence d'un usage de ses états intentionnels mentaux à titre de modèle de ceux des autres $^{13}$."

Ce qui est intéressant avec l'article de Peter Hobson, «What Puts the Jointness into Joint Attention », c'est qu'il part d'un terrain fondamentalement régi, de prime abord, par un déficit radical en termes d'attention conjointe, à savoir le cas d'enfants "avec autisme» (comme on dit aujourd'hui). Son hypothèse est de montrer que, dans ce contexte empirique, la relation à autrui emprunte des canaux qui sortent de l'alternative mentalisme/ comportementalisme. En effet, l'abord des enfants autistes révèle, une fois passée la difficulté première du contact, la création d'un lien situé en deçà du langage le plus souvent, c'est-à-dire aussi en deçà de l'échange social rationnel conventionnel. Ce type de lien est essentiellement corporel, en tout cas gestuel, et possède d'une immédiateté archaïque indéniable. Dès lors, si attention conjointe il y a, elle se situe sur ce plan organique primordial et correspond à un mode de régulation affective que l'on peut rapprocher de l'accordage (attunement) dont parle D. Stern à propos des nourrissons ou de la «résonance » en jeu dans l'approche des patients psychotiques par les psychiatres telle qu'elle est décrite par Mony Elkaïm. P. Hobson décrit quant à lui ce mode de relation en termes de relatedness ou de jointness ${ }^{14}$ et accorde une place centrale à la dimension émotionnelle de la relation ${ }^{15}$, même dans ce cas particulier propre à l'autisme où l'on est confronté à une absence d'émotion et à une focalisation sur les actes.

12. J. Call \& M. Tomasello, art. cit., p. 45-65.

13. Art. cit., p. 61.

14. Art. cit., p. 186, p. 188

15. Art. cit., p. 190-192. 


\section{Imagination et inter-personnalité : quelle phénoménologie de l'attention conjointe?}

Je voudrai pour terminer ce parcours non exhaustif me centrer sur certains points de la contribution de Johannes Roessler dans ce volume, qui me paraît être la plus en phase avec les ressources disponibles dans la tradition phénoménologique sur cette question.

Quoique l'intitulé de son article, «Joint Attention and Other Minds ${ }^{16}$ », indique une orientation marquée par la philosophie de l'esprit, l'auteur centre en réalité son propos sur le problème du mutual understanding ${ }^{17}$. C'est dire que l'attention conjointe est mise au service, en tant que mécanisme primitif, de la capacité sociale des êtres humains à se connaître les uns les autres, davantage qu'à inférer ou à imputer des croyances ou des désirs, selon les prérequis de la théorie des « autres esprits».

L'auteur développe son examen anthropologique en suivant deux fils tout à la fois complémentaires et me semble-t-il innovants : d'une part, il part de l'hypothèse selon laquelle la compréhension "from the Inside » permise par la conscience mutuelle en jeu dans l'attention conjointe suppose une re-création en soi-même, qu'il nomme "imagination", de certains aspects du point de vue de l'autre. Seule une telle «re-création » permet une intégration vécue de la texture de la conscience vécue d'autrui. À cet égard, il parle d'imaginative understanding, et parie sur une forme de transparence du vécu d'autrui à moi-même : "Lorsque vous êtes attentifs ensemble à un objet avec quelqu'un d'autre, certains aspects au moins de son expérience seront transparents pour vous ${ }^{18}$.» Quoique l'auteur ne se réfère pas explicitement à la théorie husserlienne de l'intersubjectivité en termes d'empathie, on ne peut qu'être frappé par la mise au premier plan de l'acte de transposition imaginative (Hineinphantasieren) à titre de mode d'accès au vécu d'autrui.

Le second fil conducteur, également phénoménologique, est cette fois assumé explicitement, et il sert en réalité d'alternative à la première hypothèse, de type imaginatif. À cet égard, J. Roessler prend appui sur la sociologie phénoménologique d'A. Schütz pour distinguer entre la « conscience que j'ai autrui dans une situation de face-à-face » et le cadre plus large de «l'observation sociale». L'attention conjointe s'avère un cas paradigmatique, selon l'auteur, du premier cas, ce qui tend à la situer sur le terrain d'une approche en deuxième personne et non en troisième personne, et place l'observation sociale du côté de cette dernière ${ }^{19}$.

16. J. Roessler, art. cit., in N. Eilan ed., op. cit., p. 230-259.

17. Art. cit., p. 230.

18. Art. cit., p. 231

19. Ibidem, note 1. 